\title{
Hemiarch replacement with aortic root preservation for acute type A aortic dissection
}

\author{
Andreas Habertheuer, Thomas G. Gleason, Edgar Aranda-Michel, Arman Kilic, Valentino Bianco, \\ Eric Hyzny, Zachary Kassir, Forozan Navid, Ibrahim Sultan \\ Department of Cardiothoracic Surgery, University of Pittsburgh, Pittsburgh, PA, USA \\ Contributions: (I) Conception and design: I Sultan, A Habertheuer; (II) Administrative support: I Sultan, TG Gleason, F Navid; (III) Provision of \\ study materials or patients: A Habertheuer, E Aranda-Michel, A Habertheuer, V Bianco, E Hyzny, Z Kassir, F Navid; (IV) Collection and assembly \\ of data: A Habertheuer, E Aranda-Michel, A Kilic, V Bianco, E Hyzny, Z Kassir; (V) Data analysis and interpretation: A Habertheuer, E Aranda- \\ Michel, A Kilic, V Bianco, E Hyzny, Z Kassir; (VI) Manuscript writing: All authors; (VII) Final approval of manuscript: All authors. \\ Correspondence to: Ibrahim Sultan, MD. Division of Cardiac Surgery, University of Pittsburgh, Heart and Vascular Institute, University of Pittsburgh \\ Medical Center, 5200 Centre Ave, Pittsburgh, PA 15232, USA. Email: sultani@upmc.edu.
}

\begin{abstract}
Acute Type A aortic dissection (TAAD) is a surgical emergency requiring expeditious management. The surgical principles involve restoration of true lumen blood flow with obliteration of the primary tear thereby mitigating malperfusion and restoring valvular competency. At our institution $60 \%$ of TAAD patients are treated with a hemiarch and the other $40 \%$ are treated with a total arch replacement (TAR). All cases employ the use of cerebral protection. For hemiarch replacement, retrograde cerebral perfusion (RCP), via the superior vena cava (SVC), is utilized. Antegrade cerebral perfusion is utilized via brachiocephalic vessels during a TAR. Electroencephalogram (EEG) and somatosensory evoked potentials (SSEP) are used for neurocerebral monitoring with the aim of electrical silence before circulatory arrest. Key radiographic and clinical findings are used to adjudicate between these two procedures pre and intraoperatively. Patients with any of the following undergo a TAR at our institution: (I) aortic arch aneurysm, (II) primary or re-entry tear in the aortic arch or proximal descending aorta, (III) circumferential dissection of the aortic arch, or (IV) carotid body dissection or thrombosis. Irrespective of the procedure chosen, central aortic cannulation is routinely performed via modified Seldinger technique with transesophageal echocardiography guidance. The proximal aorta is addressed while the patient is systemically cooled to hypothermia. All effort is made to retain the native aortic root and valve where possible. However, in cases where the aortic root is dilated or has a tear, a valve sparing root replacement or a modified Bentall is performed. The operative strategy for a TAAD at our institution is predicated on a tear-based paradigm. However, the extent of aortic reconstruction may be different based on surgeon and center experience.
\end{abstract}

Keywords: Acute Type A dissection; hemiarch replacement; aortic root preservation; aortic valve resuspension; deep hypothermic circulatory arrest

Received: 22 March 2020; Accepted: 20 June 2020; Published: 20 October 2021.

doi: 10.21037 /jovs-2020-26

View this article at: http://dx.doi.org/10.21037/jovs-2020-26

\section{Clinical vignette}

A 67-year-old man with a history of hypertension presented to our emergency department (ED) with acute onset sharp chest pain radiating to the back. A CT scan revealed an acute Type A aortic dissection (TAAD) with no signs of malperfusion and a small pericardial effusion. After discussions with the patient and informed consent, decision was made to take the patient to the operating room for immediate intervention. 


\section{Introduction}

There are different schools of thought on what constitutes the optimal surgical approach for acute TAAD (1-3). While the extent of aortic root and arch pathology predicates the minimal intervention necessary to restore and maintain true lumen blood flow, restore valvular competency and preserve life (i.e., tear-oriented approach in form of aortic valve resuspension and root preservation when possible along with hemiarch reconstruction), more aggressive distal procedures such as total arch replacement (TAR) with or without the use of frozen elephant trunk (FET) or classic elephant trunk (ET) technique often aim at mitigating the risk of delayed distal aneurysm formation. Extensive proximal pathology where the aortic root cannot be preserved is managed with a Bentall-type root replacement or a valve-sparing root replacement (VSRR) in selected patients, while arch aneurysms or complex distal tears with distal false lumen pressurization might necessitate some sort of TAR with distal true lumen stabilization (ET/FET). The most common procedure performed at most institutions for the management of acute TAAD is hemiarch replacement in conjunction with aortic valve resuspension to preserve the aortic root.

\section{Surgical technique}

Patients are typically brought in directly to the operating room without any delay. A radial arterial line and a central venous line are inserted in addition to large bore peripheral IVs. This is done before institution of general anesthesia to be prepared for any hemodynamic compromise. The unpredictable hemodynamic nature of TAAD with potential for fast deterioration and cardiac arrest especially with transferring the patient to the operating table and during induction of anesthesia mandates both attending cardiac surgeon and experienced anesthesiologist to be in the room at all times with a plan for rapid intervention and cannulation should hemodynamic collapse occur. Routine neurocerebral monitoring is employed throughout the operative procedure using somatosensory evoked potentials (SSEP) and electroencephalogram (EEG) assessment. Transesophageal echocardiography (TEE) is utilized to confirm the diagnosis and to evaluate baseline cardiac function including degree and etiology of aortic regurgitation.

TAAD repair is performed via median sternotomy. The extent of both proximal root and distal arch repair is predicated on (I) location of primary tear, (II) presence of any large secondary tears, (III) aneurysmal sinuses of Valsalva (SOV) and aortic arch and (IV) signs of distal malperfusion. For our patient presenting with a Debakey II TAAD and a primary tear in the proximal ascending aorta and no aneurysmal dilation of the SOV, the operation of choice was an ascending and hemiarch replacement with aortic root preservation and aortic valve resuspension.

Systemic heparin is administered after the pericardium is opened and suspended. The distal ascending aorta is cannulated directly along the lesser curvature over a wire using modified Seldinger technique (Figure 1). To achieve true lumen cannulation, the aorta is punctured with an 18 -gauge needle and a soft-tip J-wire was advanced into the true lumen of the proximal descending aorta under TEE guidance. The tract is then serially dilated and the arterial cannula is advanced over the wire with the tip placed distal to the left subclavian artery. A dual stage venous cannula is used venous return. In preparation for retrograde cerebral perfusion (RCP), the SVC is dissected, encircled with an umbilical tape, cannulated with a right-angled soft-tipped venous cannula and connected to the venous line with a Y-connector. Once the patient is on cardiopulmonary bypass $(\mathrm{CPB})$, systemic cooling is initiated to achieve EEG silence. This typically occurs between 18-22 deg C (bladder) in our experience. A retrograde cardioplegia catheter is then paced in the coronary sinus and cross clamp is applied. While retrograde cardioplegia is being administered, the aorta is transected down to the level of the sinotubular junction (STJ). The aortic valve is inspected carefully to ensure that it is free of pathology. If the aortic root is not aneurysmal and does not have a tear, it is routinely preserved. Thrombus between the adventitia and intima is removed and a piece of Teflon felt is utilized to create a 'neomedia' (4) using a sandwich technique. The adventitia and intima are then sewn to each other using running 4-0 polypropylene. The aortic valve is then resuspended at the level of the commissures using pledgeted 4-0 polypropylene sutures (Figure 2). Use of biological glues is avoided due to concerns of provoking formation of delayed pseudoaneurysms (5). The STJ is then sized with an intra-annular valve sizer and a straight woven polyester graft is anastomosed to the reconstructed aortic root using running 4-0 polypropylene sutures. Antegrade cardioplegia is then infused via the Dacron graft fulfilling multiple purposes: (I) myocardial protection, (II) evaluation of valvular competency and (III) assessment of hemostasis at the suture line. Reinforcement 


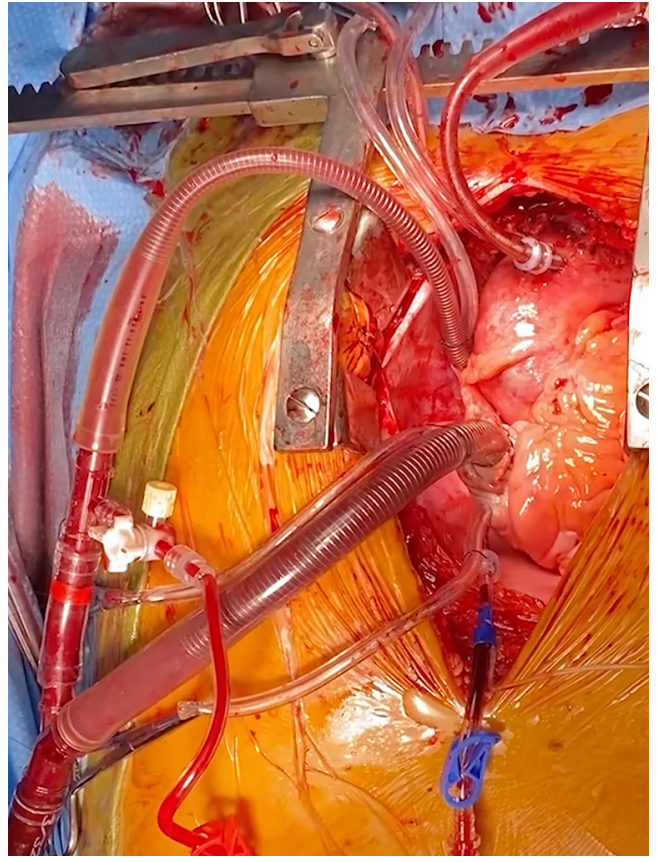

Figure 1 Cannulation strategy. Central aortic cannulation via a modified Seldinger technique under transesophageal echocardiographic guidance (TEE) is our preferred approach in the setting of acute type A aortic dissection (TAAD) and technically feasible in $>90 \%$ of patients. Contraindications to central arterial cannulation include rupture of the arch, circumferential arch dissection and complex arch tears. We routinely use a dual-staged venous cannula for the right atrium and a second right-angled softtipped venous cannula in the superior vena cava (SVC) guided into the right internal jugular vein for retrograde cerebral perfusion (RCP). Retrograde cardioplegia is administered via a coronary sinus catheter. A left ventricular vent is advanced via the right superior pulmonary vein.

sutures are placed as necessary at this point.

Once EEG silence is achieved indicating optimal core cooling, attention is shifted to the aortic arch. In preparation for RCP, the SVC is snared around the angled cannula and the side port of the internal jugular vein is transduced. CPB is discontinued and RCP is initiated with a goal SVC pressure of $26-30 \mathrm{mmHg}$. Effective RCP is confirmed both via achieving and maintaining goal venous pressures and visualization of dark blood emerging from the brachiocephalic vessels.

The aortic arch is inspected carefully to ensure no reentry tears and the aorta is trimmed to the base of the innominate artery (Figure 3). Similar to the aortic root,

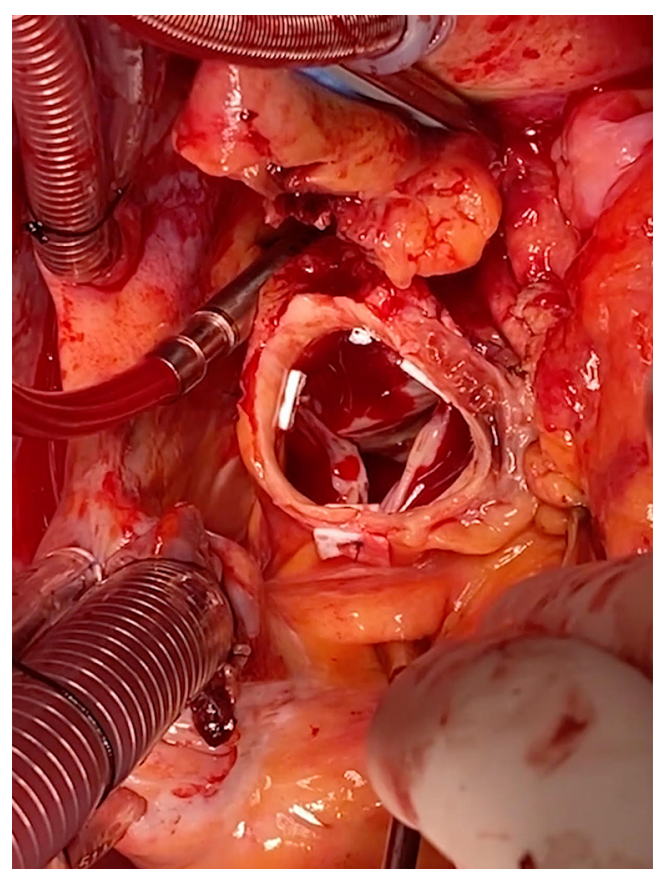

Figure 2 Proximal aortic root and valve repair. During administration of retrograde cardioplegia, the aorta is transected and trimmed to the level of the sinotubular junction (STJ), allowing inspection of the aortic root and the aortic valve. If the aortic root is non-aneurysmal and there are no intimal tears below the level of the STJ, we typically perform aortic valve suspension using pledgeted 4-0 polypropylene sutures. Retrograde medial disruptions reaching to the level of the Sinus of Valsalva are repaired by securing tailored pieces of Teflon felt between intimal and adventitia (neomedial reconstruction).

thrombus is removed from the dissection plane and intimaladventitial plane is reconstructed using the neomedial technique described above. The hemiarch anastomosis is then completed with running 4-0 polypropylene sutures using a second beveled Dacron tube graft after appropriate sizing. The graft is intussuscepted into the distal aorta to achieve intimal-graft apposition to ensure hemostasis (Figure 3). Upon completion of the anastomosis, the graft is cannulated with an arterial cannula. The brain, arch and the graft are de-aired thoroughly and the patient is placed back on $\mathrm{CPB}$ and rewarmed to normothermia. The anastomosis is inspected for and reinforced with additional sutures as needed. Both straight grafts are trimmed appropriately and beveled to mimic the natural ascending aortic curvature and the final graft-to-graft anastomosis was completed using running 2-0 polypropylene sutures (Figure 4). Hot shot 


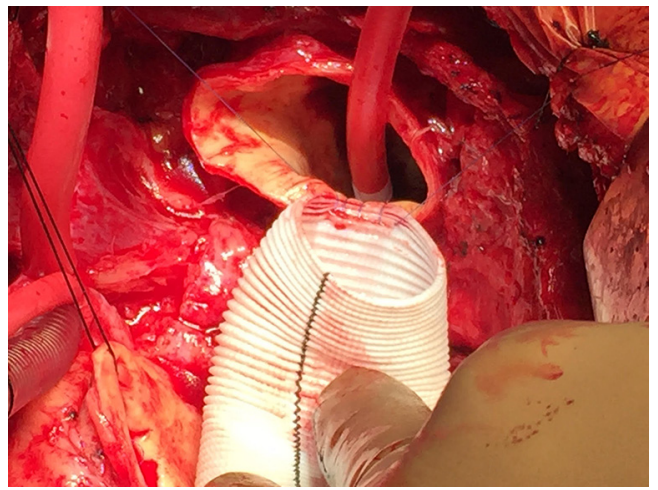

Figure 3 Distal hemiarch anastomosis. Upon confirmation of electrocerebral quiescence retrograde cerebral perfusion is initiated and maintained at a goal venous pressure of $26-30 \mathrm{mmHg}$, arterial inflow is stopped, both arterial and venous lines are clamped and the cross-clamp is opened. Following inspection of the aortic arch, neomedial reconstruction is performed by placing tailored pieces of Teflon felt into the dissected intimal-adventitial tissue planes and the dissected aortic wall is reapproximated using running 5-0 polypropylene sutures. A Dacron graft is then sized using an intra-annular valve sizer, beveled to follow the natural curvature of the aortic arch and the hemiarch anastomosis is completed by intussuscepting the graft into the aortic arch using running 4-0 polypropylene.

cardioplegia is given, the heart is de-aired and the cross clamp is released. After an adequate period of reperfusion and a period of normothermia, the heart is allowed to fill and wean off CPB. Operative approach is detailed in Video 1.

The pericardium is opened and the innominate vein identified. Central aortic cannulation is achieved via modified Seldinger technique under TEE. A dual-staged venous cannula is inserted into the right atrium for venous drainage. We use a second right-angled soft-tipped venous cannula in the SVC guided into the right internal jugular vein for RCP. Retrograde cardioplegia is administered via a coronary sinus catheter. The aorta is transected and both aortic root and aortic valve are inspected. The aortic root is non-aneurysmal with no intimal tears below the level of the STJ and decision is made to proceed with aortic valve suspension using pledgeted 4-0 polypropylene sutures. With confirmation of electrocerebral quiescence retrograde cerebral perfusion is initiated and maintained at a goal venous pressure of $26-30 \mathrm{mmHg}$ and the cross-clamp is released. The aortic arch is inspected and neomedial reconstruction with tailored pieces of Teflon felt is used to reapproximate dissected intimal-adventitial tissue

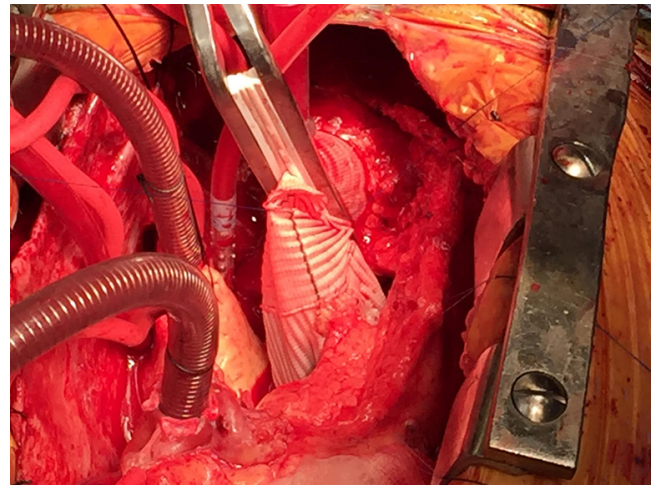

Figure 4 Graft-to-graft anastomosis. Following completion of the hemiarch anastomosis, the distal graft is cannulated, thoroughly deaired, re-clamped and cardiopulmonary bypass (CPB) is resumed. Proximal and distal Dacron grafts are then trimmed to appropriate length, beveled to follow the natural curvature of the ascending aorta and aortic continuity is established by performing a graft-tograft anastomosis using a running 3-0 polypropylene suture.

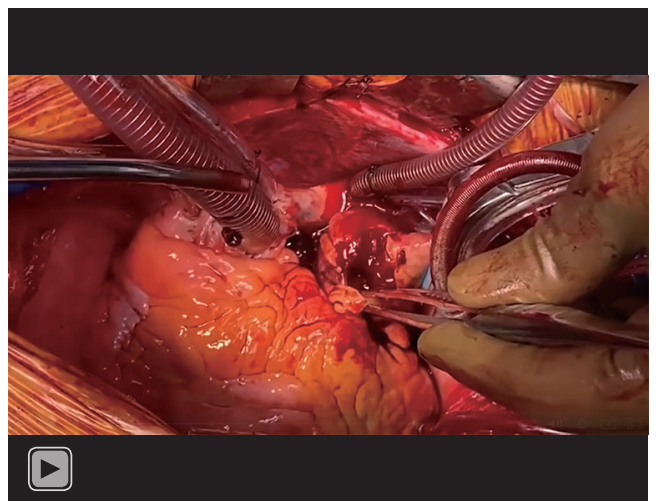

Video 1 Hemiarch replacement with aortic root preservation for acute type A aortic dissection.

planes (not shown). A Dacron graft is then sized using an intra-annular valve sizer and the hemiarch anastomosis is completed by intussuscepting the graft into the aortic arch using running 4-0 polypropylene. Following completion of the hemiarch anastomosis, the distal graft is cannulated, deaired, re-clamped and CPB is resumed. The Dacron graft is then trimmed to appropriate length and anastomosed proximally to the level of the STJ.

\section{Comment}

TAAD remains a true surgical emergency requiring fast 
and complex surgical decision making and is traditionally associated with high morbidity and mortality even in experienced hands. Proper management of patients with TAAD starts with prompt diagnosis based on CT angiogram, rapid transfer to the operating room or to a center experienced in the operative management of these patients with the goal of (I) expeditious restoration of true lumen blood flow with obliteration of false lumen flow to eliminate any malperfusion, (II) resection of the primary tear site(s) and (III) restoration of valvular competency; all while ensuring optimal neurocerebral and distal organ protection via core cooling and antegrade (ACP) and/ or RCP during circulatory arrest (6). In our experience, RCP is utilized during shorter periods of circulatory arrest (hemiarch replacement) while ACP is utilized for total arch reconstruction (7).

TAAD repair can necessitate a variety of surgical strategies from simple replacement of the ascending aorta, via some sort of aortic root reconstruction or replacement to various degrees of aortic arch reconstruction (hemiarch, zone II arch, total arch) with or without necessity of true lumen stabilization in the descending thoracic aorta via a FET or concomitant/staged antegrade (8-10) or retrograde TEVAR (11). As mortality improved over the past decade and started to plateau (12) debate has shifted to the extent of distal aortic repair, especially as long term morbidity is associated with pathologies of the arch rather than the aortic root (13) with up to $10-30 \%$ reintervention rate for the distal aorta following repair of acute TAAD (14). There have been repeated attempts at aggressive arch reconstructions beyond the hemiarch repair in this highrisk patient cohort to minimize the need for future distal reinterventions, but this needs to be balanced not only with increased risk of perioperative morbidity and mortality (15) but also with the surgeon's comfort level, particularly in low volume centers. Only isolated studies have described comparable operative mortality for patients undergoing hemiarch vs. total arch reconstruction (16). At our institution, we follow an algorithmic approach based on imaging and intraoperative findings $(6,14)$. The need for aortic arch repair (i.e., TAR) is predicated on the presence of one or more of four key findings (6): (I) presence of an aortic arch aneurysm, (II) primary, secondary or reentry tear in the arch or proximal descending aorta, (III) circumferential dissection of the aortic arch and/or (IV) carotid dissection causing cerebral malperfusion. If none of these findings are encountered, we typically proceed down the hemiarch path which is feasible in about $60 \%$ of patients in our experience. We typically perform our hemiarch cases under deep hypothermic circulatory arrest using RCP. In our experience, aggressive hemiarch replacement under deep hypothermic circulatory arrest and RCP has been associated with excellent perioperative morbidity and mortality and long term outcomes $(17,18)$. In about $40 \%$ of cases, we encounter one or more of the characteristics described above and proceed with a TAR. We routinely use ACP for our TAR cases.

If one or both carotids are dissected with presence of cerebral malperfusion and a neurologic deficit, our approach is to avoid malperfusion of the brain during circulatory arrest entirely. We achieve this by isolating the "culprit" carotid artery during the cooling phase by surgically isolating the carotid bifurcation. A trifurcate graft that is tunneled to the neck is then sewn to the carotid bifurcation and utilized for ACP (14). Prior to circulatory arrest, bilateral carotid arteries are reperfused so bilateral ACP can be utilized during circulatory arrest. The more complex surgical decision involves patients with radiographic evidence of carotid dissection without evidence of cerebral malperfusion $(14,19)$.

The aortic root is probably more challenging to regiment based on preoperative imaging studies alone (14). The decision on the type and extent of root procedure is usually established in the OR after transecting the aorta. If the intimal tear is distal to the STJ and there is some retrograde propagation of the dissection flap into the sinus that is salvageable we typically resuspend the valve at the level of the commissures and perform a neomedial root reconstruction. Even if we encounter further complicating factors such as an intimal tear proximal to the STJ, involvement of the coronary ostia or root dilation we typically try to preserve the valve in terms of a VSRR, but this is largely dependent on the surgeon's judgement, hemodynamic situation allowing longer operative times and the patient age.

Our preferred and default cannulation strategy irrespective of the extent of proximal and distal repair is central cannulation via a modified Seldinger technique and echocardiographic guidance. We are successful in centrally cannulating patients with acute TAAD in over $90 \%$ of the cases, even in cases of hemodynamic instability $(6,14)$. The surgeon should feel 2 "pops" as the needle passes through the aortic wall via the false lumen into the true lumen (20). Contraindication to central cannulation include arch rupture, circumferential arch dissection and complex arch tears at which point axillary of femoral cannulation is used (6).

In summary, contemporary management of 
patients presenting with acute TAAD entails complex multidisciplinary decision making and expeditious transfer to the operating room. In a majority of patients, a strategy of aortic root preservation with hemiarch replacement can be achieved under deep hypothermic circulatory arrest with RCP. Only distinct features require a TAR.

\section{Acknowledgments}

Funding: None.

\section{Footnote}

Provenance and Peer Review: This article was commissioned by the editorial office, Fournal of Visualized Surgery for the series "Advancement in the Surgical Treatment of Aortic Dissection". The article has undergone external peer review.

Conflicts of Interest: All authors have completed the ICMJE uniform disclosure form (available at https://jovs. amegroups.com/article/view/10.21037/jovs-2020-26/ coif). The series "Advancement in the Surgical Treatment of Aortic Dissection" was commissioned by the editorial office without any funding or sponsorship. IS served as the unpaid Guest Editor of the series and serves as an unpaid editorial board member of Fournal of Visualized Surgery from August 2019 to July 2021. AK reports personal fees from Medtronic, Inc, outside the submitted work. The authors have no other conflicts of interest to declare.

Ethical Statement: The authors are accountable for all aspects of the work in ensuring that questions related to the accuracy or integrity of any part of the work are appropriately investigated and resolved. All procedures performed in this study were in accordance with the Helsinki Declaration (as revised in 2013). The manuscript is waived from patient informed consent according to the ethics committee or institutional review board.

Open Access Statement: This is an Open Access article distributed in accordance with the Creative Commons Attribution-NonCommercial-NoDerivs 4.0 International License (CC BY-NC-ND 4.0), which permits the noncommercial replication and distribution of the article with the strict proviso that no changes or edits are made and the original work is properly cited (including links to both the formal publication through the relevant DOI and the license). See: https://creativecommons.org/licenses/by-nc-nd/4.0/.

\section{References}

1. Cohen RG, Hackmann AE, Fleischman F, et al. Type A Aortic Dissection Repair: How I Teach It. Ann Thorac Surg 2017;103:14-7.

2. Di Bartolomeo R, Leone A, Di Marco L, et al. When and how to replace the aortic arch for type A dissection. Ann Cardiothorac Surg 2016;5:383-8.

3. Leshnower BG, Chen EP. When and how to replace the aortic root in type A aortic dissection. Ann Cardiothorac Surg 2016;5:377-82.

4. Bavaria JE, Brinster DR, Gorman RC, Woo YJ, Gleason T, Pochettino A. Advances in the treatment of acute type A dissection: an integrated approach. Ann Thorac Surg. 2002;74:S1848-63; discussion S1857-63.

5. Bianco V, Kilic A, Gleason TG, et al. Management of thoracic aortic graft infections. J Card Surg 2018;33:658-65.

6. Dufendach KA, Sultan I, Gleason T. Distal Extent of Surgery for Acute Type A Aortic Dissection. Oper Tech Thorac Cardiovasc Surg 2019;24:82-102.

7. Sultan I, Bianco V, Yazji I, et al. Hemiarch Reconstruction Versus Clamped Aortic Anastomosis for Concomitant Ascending Aortic Aneurysm. Ann Thorac Surg 2018;106:750-6.

8. Sultan I. Hemiarch Replacement with Brachiocephalic endografting and Antegrade TEVAR: The New Total Arch replacement? Ann Thorac Surg 2020. [Epub ahead of print].

9. Vallabhajosyula P, Gottret JP, Menon R, et al. Central Repair With Antegrade TEVAR for Malperfusion Syndromes in Acute Debakey I Aortic Dissection. Ann Thorac Surg 2017;103:748-55.

10. Sultan I, Wallen TJ, Habertheuer A, et al. Concomitant antegrade stent grafting of the descending thoracic aorta during transverse hemiarch reconstruction for acute DeBakey I aortic dissection repair improves aortic remodeling. J Card Surg 2017;32:581-92.

11. Desai ND, Hoedt A, Wang G, et al. Simplifying aortic arch surgery: open zone 2 arch with single branched thoracic endovascular aortic repair completion. Ann Cardiothorac Surg 2018;7:351-6.

12. Parikh N, Trimarchi S, Gleason TG, et al. Changes in operative strategy for patients enrolled in the International Registry of Acute Aortic Dissection interventional cohort program. J Thorac Cardiovasc Surg 2017;153:S74-9.

13. Halstead JC, Meier M, Etz C, et al. The fate of the distal aorta after repair of acute type A aortic dissection. J 
Thorac Cardiovasc Surg 2007;133:127-35.

14. Chen E, Gleason T, Yang B, et al. Discussions in Cardiothoracic Treatment and Care: The Contemporary Management of Acute Type A Aortic Dissection. Semin Thorac Cardiovasc Surg 2020;32:202-10.

15. Sultan I, Szeto WY. Decision making in acute DeBakey I aortic dissection: Balancing extensive arch reconstruction versus mortality. J Thorac Cardiovasc Surg 2016;151:349-50.

16. Omura A, Miyahara S, Yamanaka K, et al. Early and late outcomes of repaired acute DeBakey type I aortic dissection after graft replacement. J Thorac Cardiovasc Surg 2016;151:341-8.

17. Assi R, Siki MA, Desai ND, et al. Repair of type A aortic intramural hematoma with ascending and hemiarch

doi: 10.21037/jovs-2020-26

Cite this article as: Habertheuer A, Gleason TG, Aranda-Michel E, Kilic A, Bianco V, Hyzny E, Kassir Z, Navid F, Sultan I. Hemiarch replacement with aortic root preservation for acute type A aortic dissection. J Vis Surg 2021;7:47. reconstruction using circulatory arrest and retrograde cerebral perfusion. Ann Cardiothorac Surg 2019;8:567-9.

18. Rylski B, Milewski RK, Bavaria JE, et al. Long-term results of aggressive hemiarch replacement in 534 patients with type A aortic dissection. J Thorac Cardiovasc Surg 2014;148:2981-5.

19. Sultan I, Bianco V, Patel HJ, et al. Surgery for type A aortic dissection in patients with cerebral malperfusion: Results from the International Registry of Acute Aortic Dissection. J Thorac Cardiovasc Surg 2019. [Epub ahead of print].

20. Sultan I, McGarvey J, Vallabhajosyula P, et al. Routine use of hemiarch during acute type A aortic dissection repair. Ann Cardiothorac Surg 2016;5:245-7. 